\title{
RESPUESTA HEMATOLÓGICA Y BIOQUÍMICA EN JUVENILES DE PAICHE Arapaima gigas SOMETIDOS A DIFERENTES CONCENTRACIONES DE AMONIO
}

\author{
Ingrid SÁNCHEZ ${ }^{1,2}$, Félix MEJÍA², Katty HUANUIRI², Jorge VÁSQUEZ², Anai GONZALES ${ }^{2}$, Christian \\ FERNÁNDEZ-MÉNDEZ \\ 1 Universidad Nacional de Colombia, Facultad de Medicina Veterinaria y Zootecnia. Departamento de Producción Animal, \\ Bogotá, Colombia. E-mail: iysanchezc@unal.edu.co \\ 2 Instituto de Investigaciones de la Amazonía Peruana (IIAP). Programa para el Uso y Conservación del Agua y sus \\ Recursos (AQUAREC).E-mail: cfernandez@iiap.org.pe
}

\section{RESUMEN}

El presente estudio tuvo como objetivo evaluar la respuesta hematológica y bioquímica a diferentes concentraciones de amonio de los juveniles de paiche Arapaima gigas. Evaluamos ocho tanques de 1000 litros de volumen de agua con una densidad de 5 peces $\mathrm{m}^{-3}$, que fueron sometidos a tres concentraciones de amonio: $20 \mathrm{mg} \mathrm{L}^{-1}, 15 \mathrm{mg} \mathrm{L}^{-1}, 10 \mathrm{mg} \mathrm{L}^{-1}$ y $0 \mathrm{mg} \mathrm{L}^{-1}$ (grupo control). Cuatro tanques fueron expuestos a las concentraciones de amonio por 24 horas y otros cuatro tanques por 72 horas. Los peces tuvieron un peso inicial promedio de $981,65 \pm 149,90 \mathrm{~g}$ por individuo y una longitud promedio de 50,1 $\pm 2,23 \mathrm{~cm}$. Pasado el tiempo de exposición ( 24 y 72 horas) se procedió a la extracción de $2 \mathrm{~mL}$ de sangre a cinco peces de cada concentración utilizando jeringas con anticoagulante EDTA al 10\%. Evaluamos hematocrito (Htc), conteo de eritrocitos (Eri), leucocitos (Leu) y contenido de hemoglobina $(\mathrm{Hb})$ como parámetros hematológicos y la glucosa como parámetro bioquímico. Los juveniles de paiche expuestos a 15 y $20 \mathrm{mg} \mathrm{L}^{-1}$ de amonio por 24 horas mostraron una disminución en el porcentaje de hematocrito, mientras que la glucosa mostró un incremento a $10 \mathrm{mg} \mathrm{L}^{-1}$. A las 72 horas se evidenció un aumento en el porcentaje de hematocrito en las concentraciones de 15 y $20 \mathrm{mg} \mathrm{L}^{-1}$, el resto de parámetros hematológicos (Eri, Leu. Hb, VCM, CHCM y $\mathrm{HCM}$ ) tuvieron diferentes alteraciones como respuesta a las concentraciones de amonio, sin embargo la glucosa no mostró variaciones. La exposición del paiche a diferentes concentraciones de amonio presenta variaciones hematológicas y bioquímicas que mantienen el equilibrio fisiológico de los peces.

PALABRAS CLAVE: Amazonía, pirarucú, hematología, acuicultura.

\section{HEMATOLOGICAL AND BIOCHEMICAL RESPONSE IN JUVENILES Arapaima gigas SUBJECTED DIFFERENT AMMONIUM CONCENTRATIONS}

\begin{abstract}
This study aimed to evaluate the hematological and biochemical response to different concentrations of ammonium of juveniles of Arapaima gigas. We assessed eight tanks of 1000 liters at a density of 5 fish $\mathrm{m}^{-3}$, which were exposed to three concentrations of ammonium: $20 \mathrm{mg} \mathrm{L}^{-1}, 15 \mathrm{mg} \mathrm{L}^{-1}, 10 \mathrm{mg} \mathrm{L}^{-1}$ and $0 \mathrm{mg} \mathrm{L}^{-1}$ (control group). Four tanks were exposed to ammonium concentrations for 24 hours and the other four tanks for 72 hours. The fish had an average initial weight of $981.65 \pm 149.90 \mathrm{~g}$ per individual and an average length of $50.1 \pm 2.23 \mathrm{~cm}$. After the exposure time ( 24 and 72 hours), a sample of $2 \mathrm{~mL}$ of blood was taken using syringes with anticoagulant EDTA 10\% to five fish of each concentration. We evaluated hematocrit (Htc), erythrocyte count (Eri), leucocytes (Leu) and hemoglobin content $(\mathrm{Hb})$ as hematological parameters and glucose as a biochemical parameter. The juveniles of $A$. gigas exposed to 15 and $20 \mathrm{mg} \mathrm{L}^{-1}$ of ammonium for 24 hours showed a decrease in the percentage of hematocrit, while glucose showed an increase at $10 \mathrm{mg} \mathrm{L}^{-1}$. After 72 hours, an increase in the percentage of hematocrit was observed in fish exposed to 15 and $20 \mathrm{mg} \mathrm{L}^{-1}$ of ammonium, the rest of hematological parameters (Eri, Leu, Hb, VCM, CHCM and HCM) had different alterations in response to concentrations of ammonium, and the glucose did not show variation. The exposure of $A$. gigas to different ammonium concentrations showed hematological and biochemical variations that maintain the physiological balance of the fish.
\end{abstract}

KEYWORDS: Amazon, pirarucu, hematology, aquaculture. 


\section{INTRODUCCIÓN}

Arapaima gigas paiche es uno de los peces de mayor importancia comercial en la Amazonía peruana como fuente de alimentación y generación de ingresos para los pobladores (Alcántara et al., 2006). Es considerada una especie con gran potencial de cultivo, debido a que puede tolerar altas densidades sin comprometer la supervivencia (Gonzales et al., 2016). Así mismo, el suministro de alimento balanceado en cautiverio es la principal fuente de compuestos nitrogenados en el agua, especialmente en peces carnívoros por los altos niveles de proteína que contienen (Pereira \& Mercante, 2005; Ismiño-Orbe et al., 2003) y una parte de la proteína consumida es eliminada en las excretas como nitrógeno orgánico (Baldisseroto, 2002). A gigas también tolera bajos niveles de oxígeno disuelto y altos niveles de amonio en el agua (Cavero et al., 2004; Gonzales et al., 2016), debido a que presenta respiración aérea obligatoria (SalvoSouza \& Val, 1990; Brauner \& Val, 1996), característica importante que facilitaría el cultivo intensivo a gran escala. Existen estudios que evidencian que los peces sufren diferentes alteraciones fisiológicas como estrategias para contrarrestar la toxicidad del amonio (Randall \& Tsui, 2002). Los parámetros hematológicos y bioquímicos son considerados buenos indicadores fisiológicos frente a la respuesta de estrés en peces por parámetros medioambientales o físicoquímicos, los cuales pueden alterar el patrón característico del eritrograma y bioquímica en los teleósteos (Tavares-Días \& Moraes, 2004). El presente estudio tuvo por objetivo evaluar la respuesta hematológica y bioquímica de juveniles de paiche Arapaima gigas sometidos a diferentes concentraciones de amonio.

\section{MATERIALES Y MÉTODOS}

\section{ACONDICIONAMIENTO DE LOS PECES}

Se utilizaron un total de 40 juveniles de paiche con un peso promedio de $981,65 \pm 149,90 \mathrm{~g}$ y una longitud total promedio de $50,1 \pm 2,23 \mathrm{~cm}$ por individuo, distribuidos en ocho tanques de concreto de 1000 litros con aireación constante a una densidad de 5 peces $\mathrm{m}^{-3}$ en las instalaciones del Centro de Investigaciones Fernando Alcántara Bocanegra (CIFAB) del Instituto de Investigaciones de la Amazonía Peruana (IIAP).

Los peces fueron sometidos a tres concentraciones de amonio: $10 \mathrm{mg} \mathrm{L}^{-1} ; 15 \mathrm{mg} \mathrm{L}^{-1} ; 20$ $\mathrm{mg} \mathrm{L}^{-1}$ y un grupo control $\left(0 \mathrm{mg} \mathrm{L}^{-1}\right)$ durante 24 y 72 horas, mediante la adición de cloruro de amonio $\left(\mathrm{NH}_{4} \mathrm{Cl}\right)$ para obtener las concentraciones de amonio deseadas en el agua. Se restringió la alimentación de los peces para que no influyera en las concentraciones de amonio en el agua.

Las mediciones de los parámetros físicos y químicos del agua (temperatura, oxígeno disuelto y $\mathrm{pH})$ se realizaron dos veces al día con un oxímetro (YSI, Model 55) y un medidor de $\mathrm{pH}$ (Oakton, 110 series). El amonio y los nitritos se evaluaron diariamente mediante un fotómetro colorimétrico (Hanna Instruments, HI 83203). Los valores promedios encontrados durante el experimento fueron: temperatura $\left(29,9 \pm 1,2 \mathrm{C}^{\circ}\right)$; oxígeno disuelto $\left(5,10 \pm 0,3 \mathrm{mg} \mathrm{L}^{-1}\right) ; \mathrm{pH}(6,3 \pm 0,5)$ y nitrito $(0$ $\left.\operatorname{mg~L}{ }^{-1}\right)$.

\section{COLECTA Y ANÁLISIS DE SANGRE}

Los peces fueron capturados a las 24 y 72 horas de exposición con una red de mano y ubicados en una esponja, se emplearon toallas húmedas para la inmovilización y vendaje de los ojos, el cuerpo y las branquias fueron hidratados constantemente con agua. Mediante punción de la arteria caudal a nivel del arco hemal, se extrajo $2 \mathrm{~mL}$ de sangre utilizando jeringas desechables conteniendo EDTA al 10\%; la sangre extraída fue separada en dos partes: para las muestras hematológicas se colocó la sangre en tubos de $2 \mathrm{~mL}$ conservadas en refrigeración $\left(4{ }^{\circ} \mathrm{C}\right)$ para preservar la viabilidad de las células y para las muestras bioquímicas en tubos de $0,5 \mathrm{~mL}$ que fueron centrifugados a $100 \mathrm{rpm}$ por 5 minutos para obtener el plasma sanguíneo.

Los parámetros hematológicos evaluados fueron: hematocrito (Htc) por el método del microhematocrito (Goldenforb et al., 1971) centrifugados a $100 \mathrm{rpm}$ por 5 minutos; concentración de hemoglobina $(\mathrm{Hb})$ por el método de la cianometahemoglobina (Laboratorios BIOLABTEST $^{\circledR}$ ) a $540 \mathrm{~nm}$, conteo de eritrocitos (Er) y leucocitos (Leu) con solución Natt \& Harrick (1952) a través de cámara de Neubauer y la determinación de los índices hematimétricos: volumen corpuscular medio (VCM), concentración de hemoglobina corpuscular media (CHCM) y hemoglobina corpuscular media (HCM) según el método de Wintrobe (1934). En cuanto a los parámetros bioquímicos, se determinó la concentración de glucosa (método Trinder: Laboratorios FAR Diagnstics ${ }^{\circledR}$ ) a $510 \mathrm{~nm}$. Las lecturas fueron realizadas en un espectrofotómetro de UV visible (Agilent Technologies, Cary 60. California, Estados Unidos). 


\section{ANÁLISIS DEDATOS}

Los resultados se expresaron en promedio y desviación estándar. Se utilizó el análisis de varianza (ANOVA), cuando existió diferencias significativas entre los grupos se utilizó el test de Dunnett para comparar cada tratamiento con el control usando el software estadístico "Sigma Plot 11".

\section{RESULTADOS Y DISCUSIÓN}

Los valores de los parámetros hematológicos de los juveniles de paiche encontrados en el presente estudio se muestran en la Tabla 1. Los valores promedios de hematocrito, eritrocitos, leucocitos, VCM, CHCM y HCM se encontraron dentro los valores reportados por diferentes estudios para la misma especie en condiciones de cultivo (TavaresDías et al., 2007; Menezes et al., 2006; Serrano et al., 2013, Gonzales et al., 2016; Hoshino et al., 2017). La hemoglobina es el único de los parámetros que se muestra superior a las condiciones normales, acercándose a los valores obtenidos por Ríos et al. (2015) al someter a $A$. gigas a condiciones similares al presente estudio de restricción alimentaria de los peces durante el cultivo.
A las 24 horas de exposición la mayoría de parámetros hematológicos de los juveniles de paiche (Eri, Leu, Hb, VCM, CHCM, HCM) no mostraron diferencia significativa con el control $(\mathrm{P}>0,05)$, solo el porcentaje de hematocrito tiene diferencia significativa $(\mathrm{P}<0,05)$ con el control, mostrando una disminución en las dosis más altas $\left(15\right.$ y $\left.20 \mathrm{mg} \mathrm{L}^{-1}\right)$. Este comportamiento ha sido descrito en otros peces de agua dulce cuando incrementan la concentración de amonio en el agua (El-Sherif \& El-Feky, 2008; ElSayed, 2015; Zeitoun et al., 2016). Este efecto esta asociado a tejidos como las branquias, que al ser expuestos al incremento de amonio sufren daños en el epitelio branquial (hiperplasia, fusión lamelar, hipertrofia), interfiriendo en el intercambio gaseoso y transporte iónico (Martinez et al., 2006; El-Sherif \& El-Feky, 2008) que permite la permeabilidad de la branquia desencadenando finalmente una hemodilución, sin mostrar una disminución significativa del número de eritrocitos (Eri) o el volumen de estos (VCM) que son los que influyen típicamente en el hematocrito (Htc).

Sin embargo, a las 72 horas el porcentaje de hematocrito se ha incrementado significativamente $(\mathrm{P}<0,05)$ en las dosis más altas $\left(15\right.$ y $\left.20 \mathrm{mg} \mathrm{L}^{-1}\right)$, esta

Tabla 1. Parámetros hematológicos de $A$. gigas expuestos a diferentes concentraciones de amonio durante 24 y 72 horas.

\begin{tabular}{|c|c|c|c|c|c|}
\hline \multirow{2}{*}{ Tiempo } & \multirow{2}{*}{ Parámetros } & \multicolumn{4}{|c|}{ Concentración de amonio $\left(\mathrm{mg} \mathrm{L}^{-1}\right)$} \\
\hline & & 0 & 10 & 15 & 20 \\
\hline \multirow[t]{7}{*}{$24 \mathrm{~h}$} & Htc $(\%)$ & $32,45 \pm 0,15$ & $32,58 \pm 0,05$ & $30,56 \pm 0,42^{*}$ & $30,83 \pm 0,51^{*}$ \\
\hline & $\operatorname{Eri}\left(10^{6} \mu \mathrm{L}^{-1}\right)$ & $1,69 \pm 0,21$ & $2,16 \pm 0,62$ & $2,19 \pm 0,51$ & $1,58 \pm 0,19$ \\
\hline & Leu $\left(10^{3} \mu \mathrm{L}^{-1}\right)$ & $9,74 \pm 5,53$ & $7,2 \pm 3,10$ & $7,72 \pm 3,10$ & $6,22 \pm 2,12$ \\
\hline & $\mathrm{Hb}\left(\mathrm{g} \mathrm{dL}^{-1}\right)$ & $15,49 \pm 1,01$ & $15,89 \pm 0,74$ & $16,15 \pm 2,05$ & $16,30 \pm 0,69$ \\
\hline & VCM (fL) & $194,4 \pm 23,4$ & $159,0 \pm 36,9$ & $146,3 \pm 36,4$ & $196,6 \pm 24,5$ \\
\hline & CHCM $\left(\mathrm{g} \mathrm{dL}^{-1}\right)$ & $47,7 \pm 2,93$ & $48,8 \pm 2,33$ & $52,9 \pm 6,93$ & $52,9 \pm 2,4$ \\
\hline & HCM (pg) & $92,7 \pm 11,3$ & $77,3 \pm 17,2$ & $77,1 \pm 20,7$ & $103,8 \pm 12,2$ \\
\hline \multirow[t]{7}{*}{$72 \mathrm{~h}$} & Htc $(\%)$ & $30,60 \pm 0,52$ & $33,70 \pm 0,21$ & $34,43 \pm 0,24^{*}$ & $35,03 \pm 1,16^{*}$ \\
\hline & $\operatorname{Eri}\left(10^{6} \mu \mathrm{L}^{-1}\right)$ & $1,77 \pm 0,29$ & $2,76 \pm 0,51^{*}$ & $1,50 \pm 0,10$ & $1,33 \pm 0,19$ \\
\hline & Leu $\left(10^{3} \mu \mathrm{L}^{-1}\right)$ & $3,2 \pm 0,44$ & $4,63 \pm 1,05^{*}$ & $4,0 \pm 0,28$ & $2,99 \pm 0,93$ \\
\hline & $\mathrm{Hb}\left(\mathrm{g} \mathrm{dL}^{-1}\right)$ & $14,7 \pm 0,53$ & $17,42 \pm 0,37^{*}$ & $17,35 \pm 0,73^{*}$ & $15,54 \pm 0,54$ \\
\hline & VCM (fL) & $176,3 \pm 27,3$ & $126,2 \pm 24,0^{*}$ & $229,7 \pm 20,8^{*}$ & $267,9 \pm 41,4^{*}$ \\
\hline & CHCM $\left(\mathrm{g} \mathrm{dL}^{-1}\right)$ & $48,0 \pm 1,32$ & $51,7 \pm 1,51^{*}$ & $50,4 \pm 2,81$ & $44,42 \pm 2,36^{*}$ \\
\hline & HCM (pg) & $84,5 \pm 12,2$ & $65,4 \pm 13,5$ & $115,4 \pm 6,4^{*}$ & $119,0 \pm 20,1^{*}$ \\
\hline
\end{tabular}

* Promedios significativamente diferentes del control $(\mathrm{P}<0,05)$. VCM $=$ Volumen corpuscular medio, HCM = Concentración de hemoglobina y $\mathrm{CHCM}=$ Concentración de hemoglobina corpuscular media 

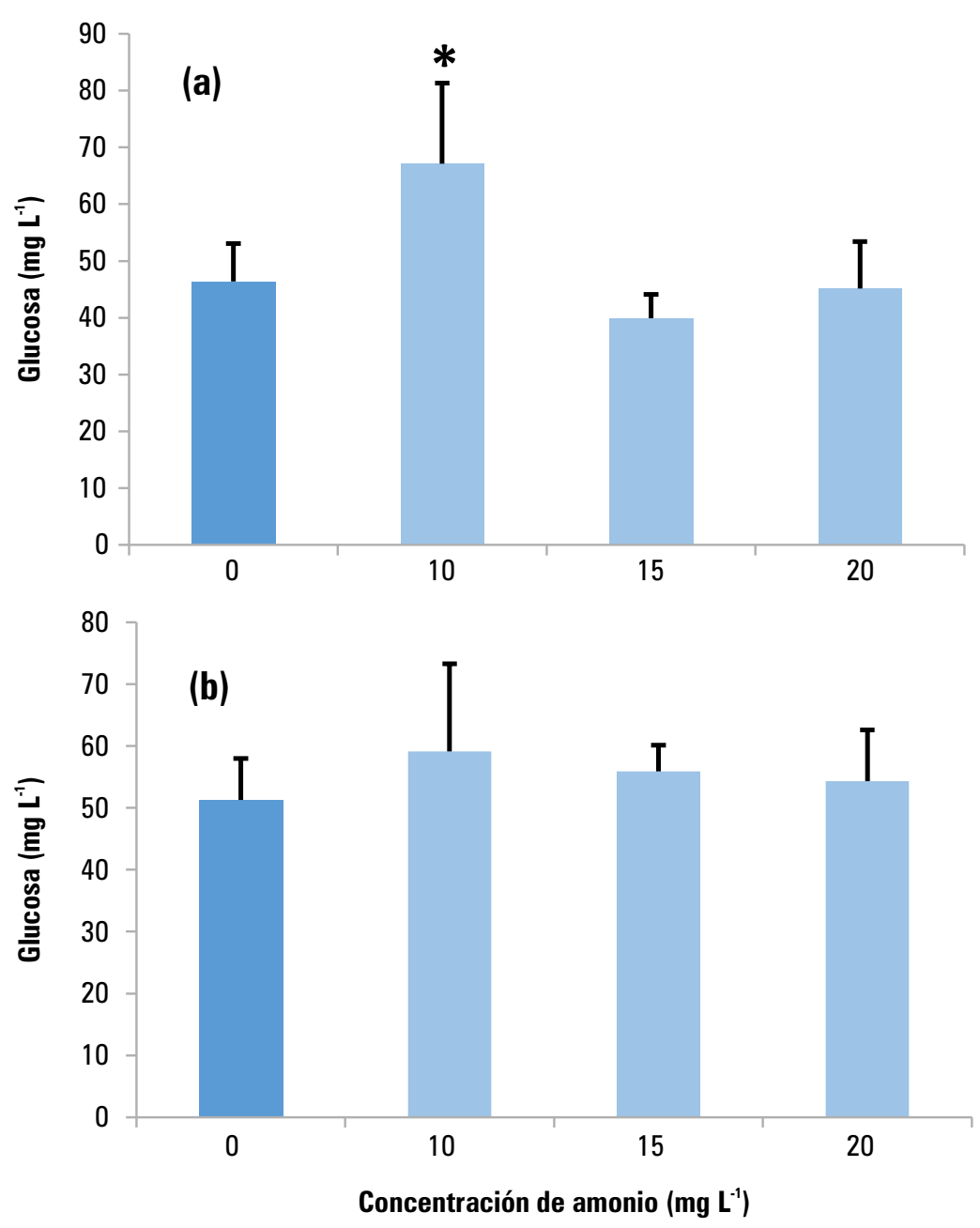

Figura 1. Concentración de glucosa en plasma de $A$. gigas expuesto a diferentes concentraciones de amonio durante (a) 24 y (b) 72 horas. * Significativamente diferente al grupo control $(P<0,05)$.

es una característica que se ha reportado cuando los peces son sometidos a estrés (Biron \& Benfey, 1994; Sopinka et al., 2016). Así mismo, la exposición a altas concentraciones en el agua de algunas sustancias (amonio, cobre, herbicida) genera estrés (Randall y Tsui, 2002; Mazon et al., 2002; Modesto \& Martinez, 2010). Estudios demuestran el incremento del hematocrito en $A$. gigas al ser sometido a estrés agudo por manipulación o transporte (Brandão et al., 2006; Gomez et al., 2006; Gomez, 2007). Los eritrocitos y leucocitos muestran un incremento significativo en la dosis menor (10 $\mathrm{mg} \mathrm{\textrm {L } ^ { - 1 }}$ ), mientras que el $\mathrm{VCM}$ se reduce significativamente a esta concentración y las dosis más altas $\left(15\right.$ y $\left.20 \mathrm{mg} \mathrm{L}^{-1}\right)$ se incrementan significativamente. El aumento del hematocrito es debido a que los eritrocitos incrementan su volumen (VCM) en las dosis mayores, pero no la cantidad de células (Er). Este incremento del volumen o tamaño indica una hinchazón de los eritrocitos como respuesta a las altas concentraciones de fármacos o sustancias en el agua debido a la toxicidad (Saravanan et al., 2011; Sharma \& Langer, 2014; Ramesh et al., 2014). El incremento de los leucocitos indica una respuesta inmune como estímulo ante la toxicidad de alguna sustancia (Saravanan et al., 2011).

Mientras que en la dosis de $10 \mathrm{mg} \mathrm{L}^{-1}$ el aumento del número de eritrocitos se equilibra con la reducción significativa del tamaño (VCM) de estos, 
confirmándose con el hematocrito que no tiene cambios significativos. El incremento de los eritrocitos sugiere que hay generación de nuevos eritrocitos inmaduros (menor tamaño) como respuesta al ser sometidos a concentraciones de sustancias subletales como el amonio (Al-Attar, 2005; Alwan et al., 2009; Alaye \& Morales, 2013; Shin et al., 2016).

La hemoglobina de los peces expuestos durante 72 horas a las concentraciones de 10 y $15 \mathrm{mg} \mathrm{L}^{-1}$ presentaron diferencia significativa $(\mathrm{P}<0,05)$ con el control. Este incremento se debe a que la hemoglobina reduce la capacidad de intercambio de oxígeno y dióxido de carbono al ser sometidos los peces a altas dosis de sustancias como el amonio (Goss \& Wood, 1988; Alwan et al., 2009; Barbieri \& Bondioli, 2015). Este incremento se ve influenciado por el aumento de la hemoglobina en cada eritrocito como lo muestra el HCM al incrementarse significativamente en las dosis mayores $(15$ y $20 \mathrm{mg}$ $\mathrm{L}^{-1}$ ) y también el incremento del CHCM en las dosis de 10 y $20 \mathrm{mg} \mathrm{L}^{-1}$. Las alteraciones de los índices hemáticos (VCM, CHCM y HCM) en las diferentes concentraciones se dan como respuesta del organismo para equilibrar los cambios en el hematocrito, hemoglobina, numero de eritrocitos y leucocitos ocasionadas por la toxicidad del amonio (Saravanan et al., 2011).

Respecto al parámetro bioquímico a las 24 horas los peces sometidos a dosis de $10 \mathrm{mg} \mathrm{L}^{-1}$ muestran un incremento de la glucosa respecto al control, mientras que a las 72 horas no hay diferencia significativa. La glucosa es un indicador útil como medida de respuesta aguda a factores de estrés específico (Martinez et al., 2006). Esta puede aumentar horas después de iniciarse el estrés, pero puede disminuir a niveles normales dentro de las 24 horas (Evans et al., 2006). Estudios en A. gigas muestran que al ocasionar un factor de estrés, los peces recuperan sus niveles normales en 24 horas o menos, manteniéndose los valores en los rangos de variación reportados (Brandão et al., 2006; Gomes, 2007). A las 24 horas la glucosa muestra variabilidad de la respuesta en los individuos sometidos a $10 \mathrm{mg}$ $\mathrm{L}^{-1}$, por lo que requeriría más horas para llegar a los niveles normales, debido probablemente a que esta baja dosis estaría actuando como un estrés crónico de lenta respuesta (Grzelak et al., 2017).

Este estudio demuestra la capacidad que tiene el paiche de soportar altas concentraciones de amonio en el agua con algunas variaciones en la respuesta hematológica y bioquímica que mantienen el equilibrio fisiológico de los peces.

\section{BIBLIOGRAFÍA CITADA}

Alcántara-Bocanegra, F.A. 2006. Observaciones sobre el comportamiento reproductivo del paiche, Arapaima gigas, en cautiverio. Folia Amazónica, 2 (1-2): 163-166.

Alaye-Rahy, N.; Morales-Palacios, J.J. 2013. Parámetros hematológicos y células sanguíneas de organismos juveniles del pescado blanco (Chirostoma estor estor) cultivados en Pátzcuaro, Michoacán, México. Hidrobiológica, 23(3): 340-347.

Al-Attar, A.M. 2005. Changes in haematological parameters of the fish, Oreochromis niloticus treated with sublethal concentration of cadmium. Pakistan Journal of Biological Sciences, 8(3): 421-424.

Alwan, S.F.; Hadi, A.A.; Shokr, A.E. 2009. Alterations in hematological parameters of fresh water fish, Tilapia zillii, exposed to aluminum. Journal of Science and its Applications, 3(1): 1219.

Baldisseroto, B. 2002. Fisiología de Peixes Aplicada a Piscicultura. Editorial Universidade Federal de Santa Maria, Santa Maria. RS. 212p

Barbieri, E.; Bondioli, A.C.V. 2015. Acute toxicity of ammonia in Pacu fish (Piaractus mesopotamicus, Holmberg, 1887) at different temperatures levels. Aquaculture Research, 46(3): 565-571.

Biron, M.; Benfey, T.J. 1994. Cortisol, glucose and hematocrit changes during acute stress, cohort sampling, and the diel cycle in diploid and triploid brook trout (Salvelinus fontinalis Mitchill). Fish physiology and Biochemistry, 13(2): 153-160.

Brandão, F.R.; de Carvalho Gomes, L.; Chagas, E. C. 2006. Respostas de estresse em pirarucu (Arapaima gigas) durante práticas de rotina em piscicultura. Acta Amazonica, 36(3): 349.

Brauner, C.J.; Val, A.L. 1996. The interaction between $\mathrm{O}_{2}$ and $\mathrm{CO}_{2}$ exchange in the obligate air breather, Arapaima gigas. And the facultative air breather, Lipossarcus pardalis. Physiology and Biochemistry of the Fishes of the Amazon, 101110 .

Cavero, B.A.S.; Pereira-Filho, M.; Bordinhon, A.M.; Da Fonseca, F.A.L.; Ituassú, D.R.; Roubach, R.; Ono, E.A. 2004. Notas Científicas Tolerância de juvenis de pirarucu ao aumento da concentração de amônia em ambiente confinado. Pesquisa Agropecuária Brasileira, 39 (5): 513 516. 
El-Sayed, A.M.S. 2015. Effect of ammonia stress on blood constitutes in Nile tilapia. Egyptian Academy Journal of Biological Sciences, 7(1): 37-44

El-Sherif, M.S.; El-Feky A. M. 2008 Effect of ammonia on Nile tilapia (O. niloticus) performance and some hematological and histological measures. Proceedings of the 8th International Symposium on Tilapia in Aquaculture, October 12-14, 2008, Cairo, Egypt, pp. 513-530.

Evans, J.J.; Pasnik, D.J.; Brill, G.C.; Klesius, P.H. 2006. Un-ionized ammonia exposure in Nile tilapia: toxicity, stress response, and susceptibility to Streptococcus agalactiae. North American Journal of Aquaculture, 68(1): 23-33.

Goldenfarb, B.; Bowyer, P.; Hall, E.; Brosious, E. 1971. Reproducibility in the hematology laboratory: the microhematocrit determination. American Journal of Clinical Pathology, 56(1), 35-39.

Gomes, L.D.C. 2007. Physiological responses of pirarucu (Arapaima gigas) to acute handling stress. Acta Amazonica, 37(4): 629-633.

Gomes, L.C.; Chagas, E.C.; Brinn, R.P.; Roubach, R.; Coppati, C.E.; Baldisserotto, B. 2006. Use of salt during transportation of air breathing pirarucu juveniles (Arapaima gigas) in plastic bags. Aquaculture, 256(1): 521-528.

Gonzales, A.; Mejía, F.; Huanuiri, K.; Sánchez, I.; Vásquez, J.; Fernández-Méndez, C. 2016. Valores hematológicos y bioquímicos de juveniles de paiche Arapaima gigas en cultivo intensivo. Folia Amazónica, 25(2): 137-144.

Goss, G.G,; Wood, C.M. 1988. The effects of acid and acid/aluminum exposure on circulating plasma cortisol levels and other blood parameters in the rainbow trout Salmo gaivdneri. Journal of Fish Biology, 32: 63-76.

Grzelak, A.K.; Davis, D.J.; Caraker, S.M.; Crim, M. J.; Spitsbergen, J.M.; Wiedmeyer, C.E. 2017. Stress Leukogram Induced by Acute and Chronic Stress in Zebrafish (Danio rerio). Comparative Medicine, 67(3): 263-269.

Hoshino, M.D.F.G.; Marinho, R.D.G.B.; Pereira, D.F.; Yoshioka, E.T.O.; Tavares-Dias, M.; Ozorio, R.O.D.A.; Rodriguez, A.F.R.; Ribeiro, R.A.; Faria, F.S.E.D.V. 2017. Hematological and biochemical responses of pirarucu (Arapaima gigas, Arapaimidae) fed with diets containing a glucomannan product derived from yeast and algae. Acta Amazonica, 47(2): 87-94.

Ismiño-Orbe, R.A.; Araujo-Lima, C.A.R.M.; Gomes, L.D.C. 2003. Excreção de amônia por tambaqui (Colossoma macropomum) de acordo com variações na temperatura da água e massa do peixe. Pesquisa Agropecuária Brasileira, 38(10): 1243-1247.

Martinez, C.B.R.; Azevedo, F.; Winkaler, E.U. 2006. Toxicidade e efeitos da amônia em peixes neotropicais. In: Cyrino, J.E.P.; Urbinati, E.C. (Eds). Tópicos especiais em biologia aquática e aqüicultura. Jaboticabal: Sociedade Brasileira de Aquicultura e Biologia Aquática. p. 81-95.

Mazon, A.F.; Monteiro, E.A.S.; Pinheiro, G.H.D.; Fernandes, M.N. 2002. Hematological and physiological changes induced by short-term exposure to copper in the freshwater fish, Prochilodus scrofa. Brazilian Journal of Biology, 62(4A): 621-631.

Pereira, L.P.F.; Mercante, C.T.J. 2005. A amônia nos sistemas de criação de peixes e seus efeitos sobre a qualidade da água. uma revisão. Boletim do Instituto de Pesca, 31(1): 81-88.

Menezes, G.C.; Tavares-Dias, M.; Ono, E.A.; Andrade, J.I.A.; Brasil, E.M.; Roubach, R.; Urbinati, E.C.; Marcon, J.L.; Affonso, E.G. 2006. The influence of dietary vitamin $\mathrm{C}$ and $\mathrm{E}$ supplementation on the physiological response of pirarucu, Arapaima gigas, in net culture. Comparative Biochemistry and Physiology. Part A, Molecular \& Integrative Physiology, 145: 274-279.

Modesto, K.A.; Martinez, C.B. 2010. Effects of Roundup Transorb on fish: hematology, antioxidant defenses and acetylcholinesterase activity. Chemosphere, 81(6): 781-787.

Natt, P.; Herrick, A. 1952. A new blood diluent for counting the erythrocytes and leucocytes of the chicken. Poultry Science, 31(4): 735-738.

Salvo-Souza, R.H.; Val, A.L. 1990. O gigante das águas doces. Ciência Hoje, 11:9-12.

Serrano, M.; Leguía, P.; Quispe, H.; Casas, V. 2013. Valores hematológicos del paiche Arapaima gigas de la Amazonía peruana. Revista de Investigaciones Veterinarias del Perú, 24(2): 248-251.

Ramesh, M.; Sankaran, M.; Veera-Gowtham, V.; Poopal, R.K. 2014. Hematological, biochemical and enzymological responses in an Indian major carp Labeo rohita induced by sublethal concentration of waterborne selenite exposure. Chemico-biological interactions, 207: 67-73.

Randall, D.J.; Tsui, T.K.N. 2002. Ammonia toxicity in fish. Marine pollution bulletin, 45(1): 17-23.

Rios, F.S.; Oba, E.T.; Fernandes, M.N.; Kalinin, A.L.; Rantin, F.T. 2005. Erythrocyte senescence and haematological changes induced by 
starvation in the neotropical fish traíra, Hoplias malabaricus (Characiformes, Erythrinidae). Comparative Biochemistry and Physiology, Part A, 140(3): 281-287.

Saravanan, M.; Karthika, S.; Malarvizhi A.; Ramesh, M. 2011. Ecotoxicological impacts of clofibric acid and diclofenac in common carp (Cyprinus carpio) fingerlings: Hematological, biochemical, ionoregulatory and enzymological responses. Journal of Hazardous Materials, 195:188-194

Sharma, J.; Langer, S. 2014. Effect of manganese on haematological parameters of fish, Garra gotyla gotyla. Journal of Entomology and Zoology Studies, 2(3): 77-81.

Shin, K.W.; Kim, S.H.; Kim, J.H.; Hwang, S.D.; Kang, J.C. 2016. Toxic effects of ammonia exposure on growth performance, hematological parameters, and plasma components in rockfish, Sebastes schlegelii, during thermal stress. Fisheries and Aquatic Sciences, 19(1): 44.

Sopinka, N.M.; Donaldson, M.R.; O'Connor, C.M.; Suski, C.D.; Cooke, S.J. 2016. Stress indicators in fish. Biology of Stress in Fish, 35: 406-436.
Tavares-Días, M.; De Moraes, F.R. 2004. Hematología de peixes teleósteos. 144pp.

Tavares-Dias, M.; Barcellos, J.F.M.; Marcon, J.L.; Menezes, G.; Ono, E.A.; Affonso, E.G. 2007. Hematological and biochemical parameters for the pirarucu Arapaima gigas Schinz, 1822 (Osteoglossiformes, Arapaimatidae) in net cage culture. Electronic journal of ichthyology, 2: 6168.

Wintrobe, M. 1934. Variations on the size and haemoglobin content of erythrocytes in the blood various vertebrates. Folia Haematologica, 51: 32-49

Zeitoun, M.M.; EL-Azrak, K.E.D.M.; Zaki, M.A.; Nemat-Allah, B.R.; Mehana, E.S.E. 2016. Effects of ammonia toxicity on growth performance, cortisol, glucose and hematological response of Nile Tilapia (Oreochromis niloticus). Aceh Journal of Animal Science, 1(1):21-28.

Recibido: 14 de Febrero del 2017

Aceptado para publicación: 18 de Abril del 2017 
